\title{
EXPERIENCIA INICIAL EN EL DIAGNÓSTICO Y TRATAMIENTO DEL TUMOR VESICAL SUPERFICIAL CON HEXVIX®
}

\author{
José M. Abascal Junquera, Miguel Hevia Suárez, José M. Abascal García, Cristina Estébanez, \\ Aurora Astudillo' y Ramón Abascal.
}

Servicios de Urología ll y Anatomía Patológica'. Hospital Universitario Central de Asturias. Oviedo. España.

\begin{abstract}
Resumen.- OBJETIVO: El actual standar en el diagnóstico y seguimiento del tumor vesical es la cistoscopia con luz blanca. Recientes estudios sugieren que la cistoscopia con fluorescencia a través de derivados de las porfirinas puede mejorar la detección de lesiones vesicales. Hemos analizado los resultados en el diagnóstico y tratamiento de lesiones vesicales utilizando la cistoscopia de fluorescencia con hexaminolevulinato (Hexvix®) en nuestro centro.
\end{abstract}

MÉTODOS: Entre septiembre 2006 y septiembre 2007, a 39 pacientes consecutivos con sospecha ó confirmación de tumor vesical se les administró una instilación endovesical de Hexvix® una hora antes de la cirugía. Se realizó en primer lugar una cistoscopia con luz blanca (CLB) y posteriormente con luz azul (CLA). Se resecaron todas las lesiones papilares y las sospe- chosas para análisis histológico. La edad media fue de 70.1 años (50-86). 30 hombres (76.9\%) y 9 mujeres (23. $1 \%$ ). Las características de los tumores fueron: $18 \%$ tumores primarios, $51 \%$ recurrencias y $30 \%$ controles cistoscópicos. 24 pacientes habían recibido con anterioridad algún tratamiento (9 mitomicina C y 15 BCG). 7 pacientes demostraron citologías urinarias positivas previas.

RESULTADOS: Todas las lesiones papilares visualizadas con luz blanca se confirmaron con luz azul 18 pacientes). De éstos, 17 tuvieron biopsias positivas $16 \mathrm{pTaG} 1$, 9 pTIG 1-3, 1 pT2, 1 CIS). En 15 pacientes (38.4\%) se objetivó al menos 1 lesión más solo visualizada con la luz azul. En este grupo, 8 pacientes (20.5\%) tuvieron un diagnóstico histológico positivo (3 pTaG 1, 2 pTlG3, 3 CIS). En 5 de estos casos (13\%) el manejo terapéutico posterior cambió al introducir la luz azul (BCG vs MMC). Todos los casos de CIS (4) fueron diagnosticados mediante luz azul. En el postoperatorio no se demostraron efectos secundarios (locales ó sistémicos) debidos a la administración de Hexvix®.

CONCLUSIONES: Nuestros resultados sugieren un incremento en el diagnóstico de lesiones tumorales vesicales, papilares y planas, con el uso de la cistoscopia bajo fluorescencia. Esto ha determinado un cambio de actitud terapéutica relevante en un 13\% de los pacientes. Obviamente, se necesita mayor experiencia para consolidar estos resultados y un seguimiento a largo plazo para valorar el impacto a nivel de recurrencia y progresión tumoral.

Palabras clave: Tumor vesical superficial. Diagnóstico. Hexvix $\circledR_{\text {. }}$. 
Summary.- OBJECTIVES: White light cystoscopy is the current standard for the diagnosis of bladder cancer and monitorization for recurren-ce. Recent studies suggest that porphyrin based fluorescence cystoscopy may im-prove endoscopic detection of bladder tumors. We aimed to evaluate the improvement that hexaminolevulinate fluorescence cystoscopy could lead in bladder cancer detection and treatment at one single centre.

METHODS: Between September 2006 and September 2007 a total of 39 patients with known or suspected bladder cancer underwent bladder instillation with $50 \mathrm{ml} 8 \mathrm{mM}$ hexaminolevulinate (HAL) for 1 hour. The bladder was inspected using white light cystoscopy (WLC), followed by blue light (fluorescence) cystoscopy (BLC). Papillary and suspicious lesions were resected for histological examination. Mean age was 70.1 years (50-86). Thirty patients were male (76.9\%) and 9 female (23. $1 \%)$. The tumor characteristics were: $18 \%$ primary tumors, $51 \%$ recurrences and 30\% control cystoscopies. 24 patients had previously received some treatment 19 MMC and 15 BCG). Only 7 patients had previous positive urine cytology.

RESULTS: All papillary lesions visualized with WLC were confirmed by BLC (18 patients). From these, 17 have positive biopsies (6 pTaG1, 9 pTI G 1-3, 1 pT2, 1 CIS). In 15 patients (38.4\%) we found at least 7 lesion more with BLC. In this group 8 cases (20.5\%) had positive histological diagnosis (3 pTaG1, 2 pTIG3, 3 CIS). In five patients (13\%) post-TUR therapeutic management has changed by using BLC (BCG vs MMC). All four patients with CIS were diagnosed by BLC. There was no evidence of local or systemic side effects due to HAL in the postoperative time.

CONCLUSIONS: Our results suggest there is an im-provement in the diagnosis of papillary and flat lesions in bladder cancer by using HAL fluorescence cystoscopy. This has changed the management in the postoperative period (MMC vs BCG) in $13 \%$ of the patients. Obviously, we need more patients to assess our data and long term follow-up to analyze the impact in terms of tumor recurrence and progression.

Keywords: Superficial bladder cancer. Diagnosis. Hexvix®.

\section{INTRODUCCIÓN}

El tumor vesical representa la cuarta neoplasia más frecuente en hombres y la octava en mujeres (1), significando 120000 nuevos casos en Europa cada año (2). En un $70 \%$ de los casos se presenta como tumor superficial, siendo característica su alta tasa de recurrencia (50-75\%) (3). El actual estándar en el diagnóstico y seguimiento del tumor vesical sigue siendo la cistoscopia con luz blanca (CLB) y las citologías urinarias, aunque se reconoce que la CLB no es un método adecuado para la detección de lesiones planas como el carcinoma in situ (CIS), lesión agresiva con un riesgo de progresión importante (3).

En los últimos años se viene desarrollando en Urología el diagnóstico fotodinámico con el propósito de mejorar la detección de lesiones planas ó incluso pequeños tumores papilares que pasan desapercibidos en la CLB. Este método podría tener repercusiones a nivel de la progresión tumoral al aumentar el diagnóstico de lesiones agresivas con mayor precocidad y disminuir la tasa de recurrencias (4).

La experiencia clínica en el diagnóstico del tumor vesical por fluorescencia comenzó en los años 60 con el uso de tetracicilinas (5). Posteriormente en los años 80, se utilizaron el azul de metileno (6) y los derivados de las porfirinas por vía endovenosa (7). En los años 90, encabezados por el grupo de Munich del profesor A. Hofstetter, surgieron las primeras experiencias sobre el 5-alfa-aminolevulínico (5-ALA) (8), precursor natural de la protoporfirina IX (figura 1). El excesivo aporte exógeno de 5-ALA conlleva a un acúmulo temporal de la protoporfirina IX (PpIX) a nivel mitocondrial, que es un potente fotosensibilizador. Además parece que este acúmulo de PpIX ocurre preferentemente a nivel de las células tumorales ó con gran proliferación (pretumorales) (9). En la actualidad existen ya estudios prospectivos randomizados en los que tras 5 y 8 años de seguimiento se demuestra una disminución de la tasa de recurrencias en pacientes sometidos a resección transuretral de vejiga con 5 ALA frente a la resección convencional $(10,11)$.

También la hypericina ha sido descrita como fotosensibilizador para el diagnóstico del tumor vesical. Se trata de un derivado natural del Hypericum perforatum ("flor de San Juan") utilizado como antidepresivo. Todavía existe poca experiencia clínica con su administración endovesical en este campo $(12,13)$, pero parece que la hypericina mejora la especificidad del 5-ALA a la hora de diagnosticar lesiones malignas y logra una mejor penetración en el urotelio (4).

Recientemente se ha desarrollado un derivado ester del 5-ALA, el hexylester hexaminolevulinato (Hexvix $\AA$, GE Healthcare, Londres), con objeto de mejorar la baja liposolubilidad del 5-ALA y por tanto, su baja capacidad de penetración en los tejidos. El hexaminolevulinato ha demostrado tras su instilación endovesical mayor penetración en el urotelio y una concentración más alta de PpIX, que el 5-ALA (14). 
Para la utilización del Hexvix $\AA$ se realiza una instilación endovesical 1-2 horas antes de la intervención, en una proporción de $85 \mathrm{mg}$ disueltos en $50 \mathrm{ml}$ de disolvente. No es necesaria la retención de la solución hasta el momento de la exploración. El sistema de fotodetección consta de una luz lámpara de xenón, con un filtro que permite realizar la cistoscopia con ambas luces mediante un interruptor de pedal. La PpIX absorbe fotones a $400 \mathrm{~nm}$ color azul y emite fotones (fluorescencia) a $635 \mathrm{~nm}$ de color rojo. Esto hace que podamos observar el contraste entre el fondo azul vesical, y las zonas lesionadas en rojo (fluorescencia). Asimismo, para la exploración fotodinámica son necesarias una óptica especial y una cámara de 3 chips (15).

Desde la aprobación del Hexvix® en septiembre de 2006 en 26 países europeos, incluido España, se nos presenta una nueva herramienta de utilidad para el manejo de los tumores vesicales. En este trabajo presentamos nuestra experiencia inicial con el Hexvix ${ }^{\circledR}$ en el manejo de los tumores vesicales superficiales.

\section{MATERIAL Y MÉTODOS}

Entre septiembre de 2006 y septiembre 2007 se han incluido 39 pacientes con sospecha ó confirmación de tumor vesical, de manera consecutiva en

TABLA I. CARACTERÍSTICAS GENERALES DE LA POBLACIÓN A ESTUDIO. (39 PACIENTES).

\begin{tabular}{|c|c|}
\hline Edad (años) & $70,1(50-86)$ \\
\hline Sexo (o\%) & $30(77 \%) / 9(23 \%)$ \\
\hline Tipo de tumor & $\begin{array}{c}\text { Primario }(17,9 \%) \\
\text { Recidiva }(51,3 \%) \\
\text { Control }(30,8 \%)\end{array}$ \\
\hline Tratamiento previo & $\begin{array}{c}\text { Ninguno }(38,5 \%) \\
\text { BCG }(38,5 \%) \\
\text { Mit C }(23,1 \%)\end{array}$ \\
\hline Citologías urinarias & $\begin{array}{c}\text { Ausente }(64 \%) \\
\text { Negativa }(16 \%)\end{array}$ \\
& Positiva (20\%) \\
\hline
\end{tabular}

nuestro centro. No se realizó randomización alguna de los pacientes puesto que se procedió a realizar la cistoscopia con luz azul (CLA) tras la cistoscopia estándar de manera sistemática.

Se excluyeron del estudio todos los pacientes con hematuria, infección activa, embarazo, porfiria y aquéllos con instilación de BCG $\mathrm{u}$ otros quimioterápicos endovesicales, durante las 6 semanas previas a la cirugía. La edad media fue de 70.1 años (50-86). 30 hombres $(76.9 \%)$ y 9 mujeres $(23.1 \%)$. Las características de los tumores fueron: $18 \%$ tumores primarios (7 pacientes), $51 \%$ recurrencias (20) y $30 \%$ controles cistoscópicos (12); 24 pacientes habían recibido con anterioridad algún tratamiento endovesical con más de seis semanas de antelación $(9$ mitomicina $C$ y 15 BCG). 7 pacientes demostraron citologías urinarias positivas previas (Tabla I).

A todos los pacientes se les realizó una instilación endovesical de Hexvix ${ }^{\circledR}$ entre 1 y 2 horas antes de la cirugía $185 \mathrm{mg}$ disueltos en $50 \mathrm{ml}$ de disolvente). Para realizar tanto la CLB como la CLA, utilizamos el mismo equipo endoscópico de la casa Storz (Kart Storz, Tuttlingen, Alemania), que permite inspeccionar la vejiga bajo luz blanca y fluorescencia mediante un interruptor de pedal. El equipo consta de una fuente de luz y una óptica de $30^{\circ}$ especiales. Hemos utilizado una cámara de tres chips de la misma casa comercial. Se realizó en primer lugar una cistoscopia estándar y posteriormente mediante fluorescencia. Primeramente se biopsiaron las lesiones demostrables sólo con luz azul. Posteriormente se resecaron todas las lesiones papilares visibles con la luz estándar. Se evaluó por cada paciente el número de lesiones diagnosticadas y su resultado histopato-

\section{TABLA II. RESUMEN DE LAS LESIONES SOSPECHA- DAS EN FUNCIÓN DEL TIPO DE DIAGNÓSTICO ICLB: CISTOSCOPIA CON LUZ BLANCA; CLA: CISTOSCOPIA CON LUZ AZUL; CLAB: LESIÓN VISUALIZADA CON AMBASI.}

\begin{tabular}{|c|c|c|}
\hline \multicolumn{3}{|c|}{ Diagnóstico de lesiones sospechosas } \\
\hline & № PACIENTES (\%) & № LESIONES \\
\hline CLB & 0 & 0 \\
\hline CLA & $15(38)$ & 23 \\
\hline CLAB & $18(46)$ & 31 \\
\hline
\end{tabular}




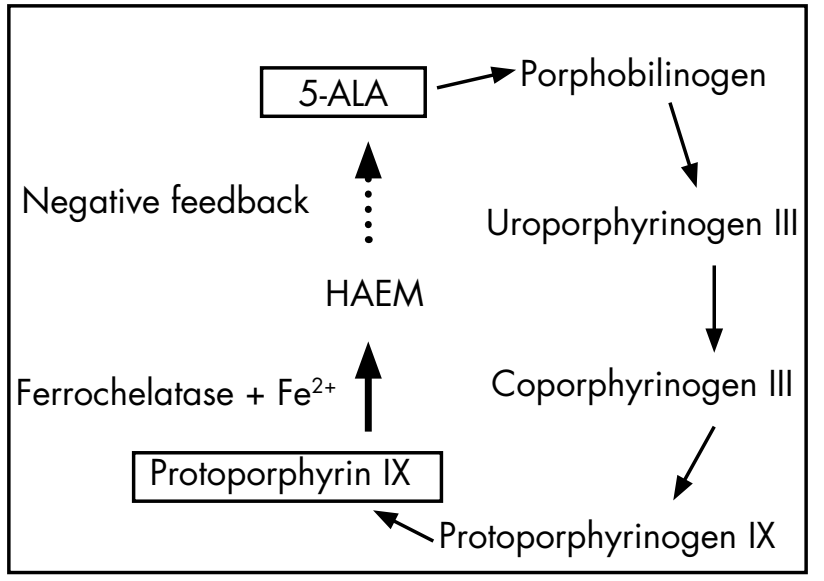

FIGURA 1. Representación esquemática de la vía de biosíntesis del grupo hemo. El 5-ALA es un precursor natural de la protoporfirina IX que precede a la formación del grupo hemo, paso esencial en el metabolismo mitocondrial.
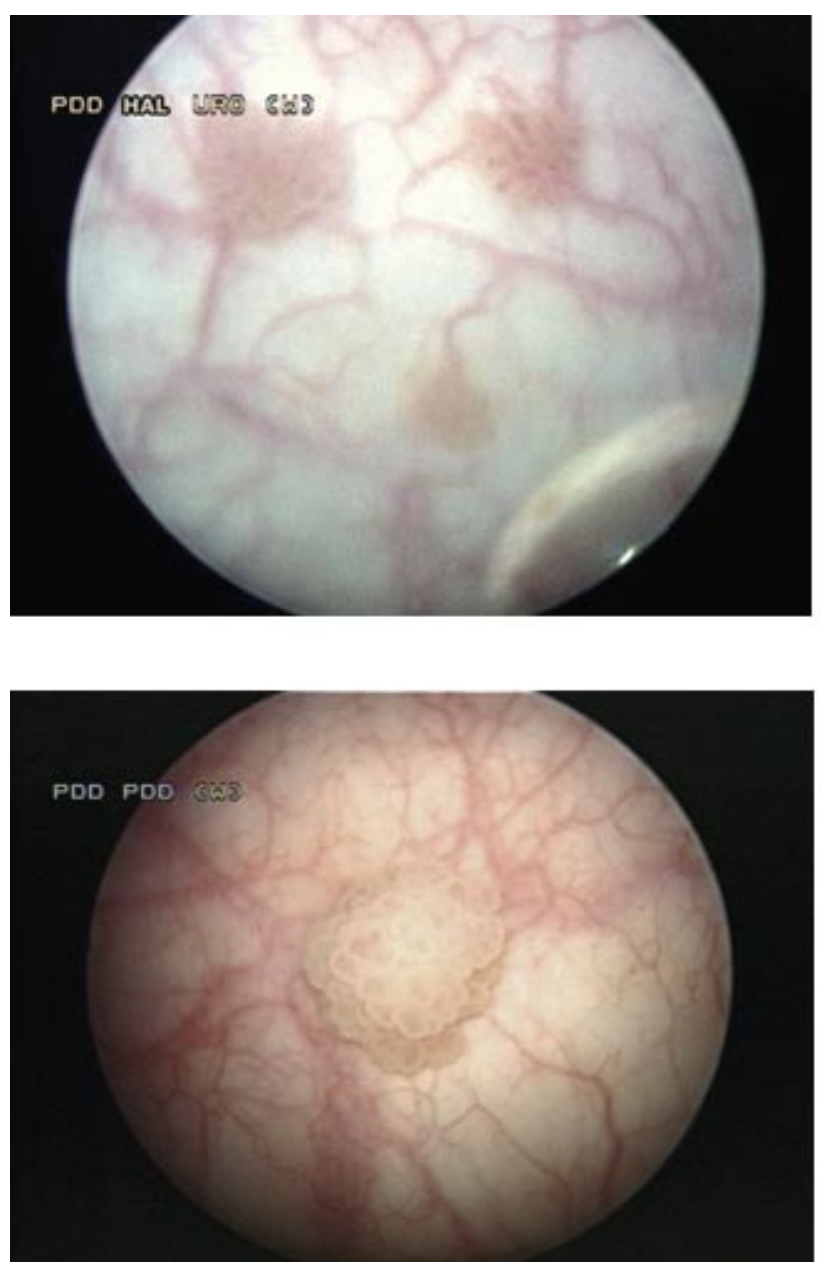

lógico, sólo con la luz blanca, sólo con luz azul y lesiones visibles con ambas (Figuras 2 y 3 ).

Durante las 72 horas posteriores a la instilación endovesical del Hexvix $₫$, se evaluaron efectos secundarios locales ó generales debidos directamente al producto.

\section{RESULTADOS}

Todos los pacientes fueron clasificados en función del modo de diagnóstico de la lesión: sólo con cistoscopia estándar (CLB), sólo con cistoscopia bajo fluorescencia (CLA) ó bajo ambas (CLAB).

En 15 de los 39 pacientes (38\%) se biopsiaron lesiones con CLA; en 7 casos de este subgrupo se trataba de una sola lesión sospechosa; en 8 , se tomaron 2 ó más biopsias. En 18 de los 39 pacientes
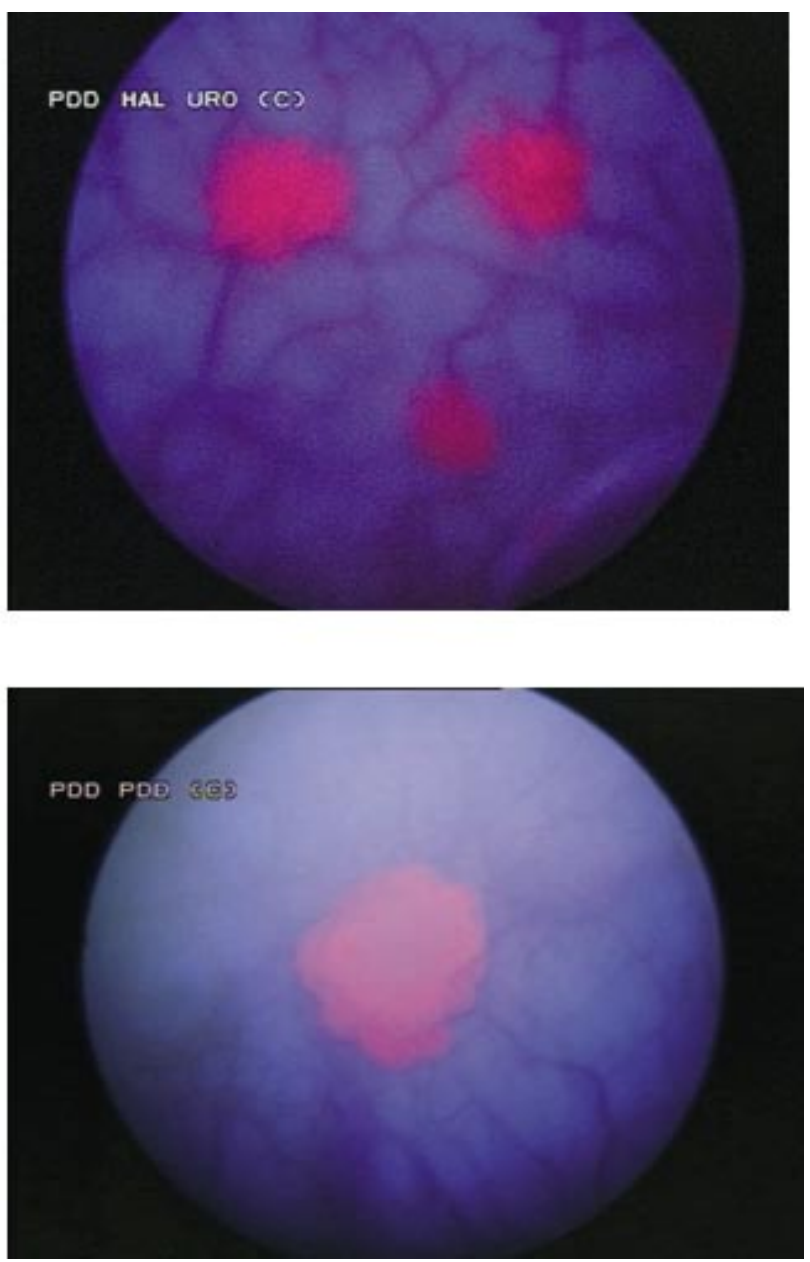

FIGURA 2. Ejemplos endoscópicos de lesiones visibles con luz blanca y confirmadas por la emisión de fluorescencia roja con la luz azul. 
(46\%) el diagnóstico se hizo bajo CLAB; de entre éstos, 5 tenían una sola lesión sospechosa y 13, más de una. No hubo ningún caso en el que se tomasen biopsias solo con CLB, es decir, que todas las lesiones papilares observadas con la cistoscopia estándar se confirmaron mediante fluorescencia, pero que además en 15 casos se tomaron muestras de lesiones sospechosas sólo con la cistoscopia bajo fluorescencia (Tabla II).

El resultado anatomopatológico se resume en las Tablas III y IV, en función de los pacientes biopsiados y el número de lesiones. De los 15 pacientes en los que se realizaron biopsias mediante CLA, fueron positivas en 8 (53\%) (3 pTaG1, 2 pTIG 1-3, $3 \mathrm{CIS})$. De los 18 pacientes en que se tomaron biop-
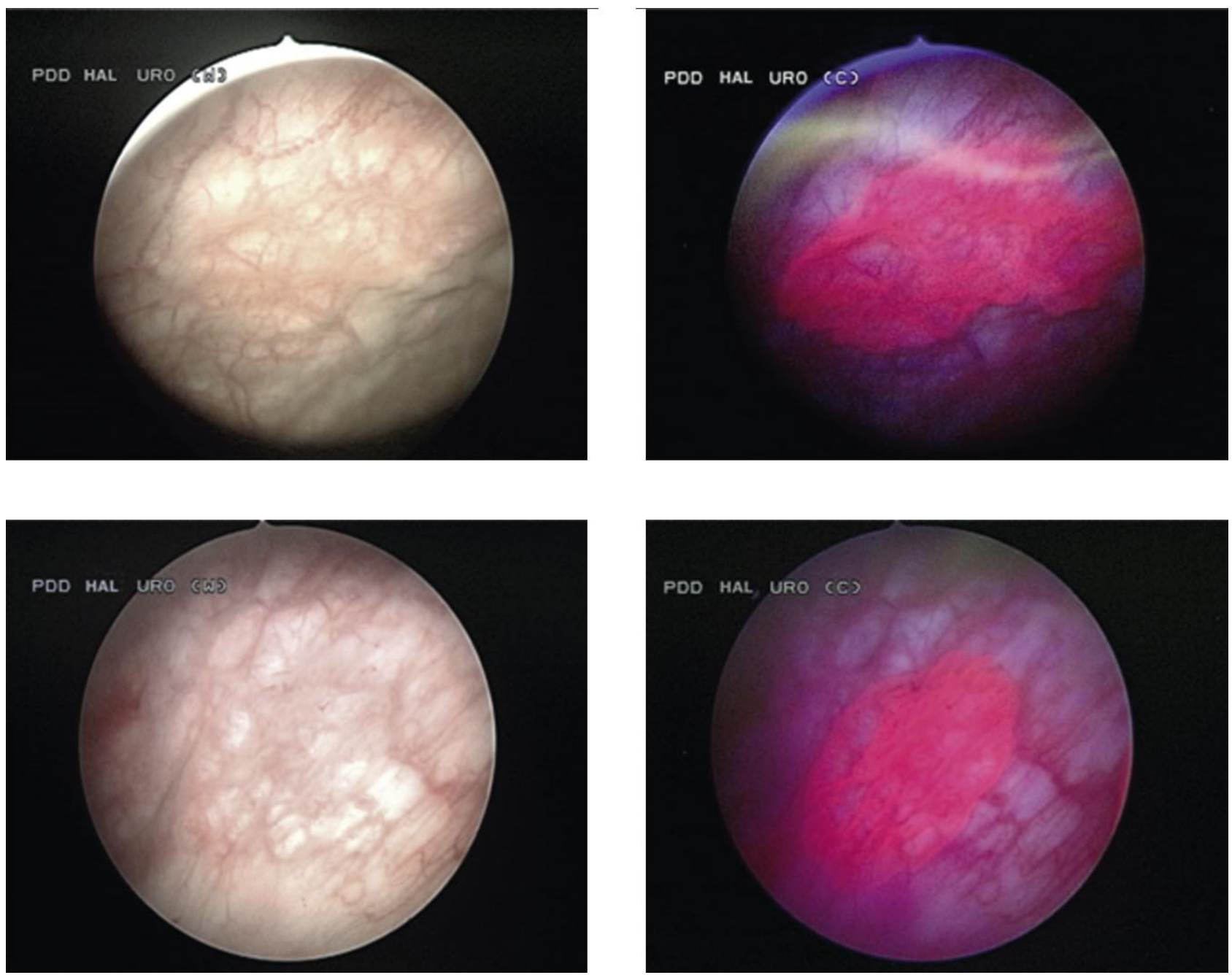

FIGURA 3. Ejemplos endoscópicos de lesiones sólo visibles con luz azul: a la izquierda, cistoscopia con luz estándar en la que no se aprecian claramente lesiones sospechosas; a la derecha, aparición de lesiones con fluorescencia (en este caso, carcinoma in situ). 
repetidamente, en 6 casos negativas y 26 pacientes no las tenían. De los 7 pacientes con citologías " + ", se recogieron biopsias mediante CLA en 4 , resultando positivas en el $75 \%(2, \mathrm{ClS} ; 1$, pTa). Los otros 3 pacientes en los que por tener citologías positivas y ninguna lesión visible con CLB ni con CLA se realizó una biopsia aleatorizada, que resultó negativa en todos los casos.

Analizando el tratamiento que se adjudicó a cada paciente tras evaluar el resultado anatomopatológico de las biopsias realizadas en función del método de detección, observamos que en 5 de los 39 pacientes $(13 \%)$ hubo un cambio en el tratamiento adyuvante debido a lesiones diagnosticadas de más por la cistoscopia con fluorescencia. En concreto, estos pacientes iniciaron tratamiento con $B C G$ en vez de mitomicina $C$, en función del protocolo actual de nuestro servicio.

Durante las 72 horas posteriores a la instilación endovesical del Hexvix $®$ no se registraron eventos adversos que pudiesen relacionarse directamente con la administración del producto.

\section{DISCUSIÓN}

Uno de los principales puntos controvertidos en el manejo del tumor vesical superficial es el alto índice de recurrencias. En parte debido a resecciones incompletas ó por lesiones que pasan desapercibidas durante la resección endoscópica. Esto puede implicar además un aumento en el riesgo de progresión de estos tumores, al dejarse de diagnosticar lesiones como el carcinoma in situ.

TABLA III. RESULTADO ANATOMOPATOLÓGICO EN FUNCIÓN DEL NÚMERO DE PACIENTES BIOPSIADOS.

\begin{tabular}{|c|c|c|}
\hline \multicolumn{3}{|c|}{$\begin{array}{c}\text { Resultado anatomopatológico de } \\
\text { lesiones sospechosas }\end{array}$} \\
\hline & $\begin{array}{c}\text { No PACIENTES (\%) } \\
\text { con lesión + } \\
\text { /total pacientes } \\
\text { biopsiados }\end{array}$ & AP no pacientes \\
\hline CLB & 0 & 0 \\
\hline CLA & $15(38)$ & 23 \\
\hline CLAB & $18(46)$ & 31 \\
\hline
\end{tabular}

En este trabajo se evalúa la aportación de la fotodinamia en el diagnóstico y tratamiento de los tumores vesicales. En los últimos años se han venido publicando varios trabajos en los que se demuestra un claro aumento del diagnóstico de tumores vesicales papilares y del carcinoma in situ con el 5-ALA (16) y con el Hexvix® (17-21).

En la actualidad existen ya estudios prospectivos randomizados en los que tras 5 y 8 años de seguimiento se objetiva una disminución de la tasa de recurrencias en pacientes sometidos a resección transuretral de vejiga con 5-ALA frente a la resección convencional $(10,11)$.

En nuestra serie en un $20,5 \%$ de los pacientes se resecó al menos una lesión patológica más, de lo que hubiésemos obtenido con la resección estándar bajo luz blanca. Esto se tradujo a corto plazo en un beneficio por el cambio de indicación del tratamiento adyuvante en el $13 \%$ de los casos; además, en el resto de pacientes hubiese significado una recidiva segura en el primer control cistoscópico, por lo que también en este sentido salen beneficiados. En el trabajo de Jocham D. y cols. (19), el aumento de lesiones tumorales diagnosticadas mediante Hexvix $\circledR_{\text {, }}$ se tradujo en un cambio del tratamiento posterior a la resección en el $17 \%$ de los pacientes.

En esta misma línea, Schmidbaver J. y cols. (18) mejoran las tasas de detección con Hexvix ${ }^{\circledR}$ frente a la cistoscopia estándar de tumores papilares pTa $(97 \%$ vs $88 \%)$ y del carcinoma in situ $197 \%$ vs $58 \%$ ). En un estudio reciente en el que se evaluaron 311 pacientes (20), la cistoscopia bajo fluorescencia detectó al menos 1 lesión más pTa en el $29 \%$ de los

TABLA IV. RESULTADOO ANATOMOPATOLÓGICO EN FUNCIÓN DEL NÚMERO DE LESIONES BIOPSIADAS.

\begin{tabular}{|c|c|c|}
\hline \multicolumn{3}{|c|}{$\begin{array}{c}\text { Resultado anatomopatológico de } \\
\text { lesiones sospechosas }\end{array}$} \\
\hline & $\begin{array}{c}\text { № lesiones +/total } \\
\text { lesiones biopsiadas (\%) }\end{array}$ & AP no pacientes \\
\hline CLB & 0 & 0 \\
\hline CLA & $12 / 23(52)$ & pTa (5), pT1 (4), CIS (3) \\
\hline CLAB & $29 / 31$ (93) & pTa (14), pT1 (13), \\
& & pT2 (1), CIS (1) \\
\hline
\end{tabular}


TABLA V. PACIENTES DIAGNOSTICADOS DE CARCINOMA IN SITU EN FUNCIÓN DEL MÉTODO

\begin{tabular}{|c|c|}
\hline \multicolumn{2}{|c|}{ Diagnóstico CIS } \\
\hline & № PACIENTES (\%) \\
\hline CLB & 0 \\
\hline CLA & $3(75)$ \\
\hline CLAB & $1(25)$ \\
\hline
\end{tabular}
DIAGNÓSTICO

está exenta de falsos positivos en la toma de biopsias, describiéndose tasas de hasta el $90 \%(22)$; con el uso del Hexvix®, no consideramos necesarias las biopsias múltiples randomizadas, siendo suficiente con biopsiar aquellas zonas que emiten fluorescencia.

En definitiva pensamos que esta nueva herramienta diagnóstica y terapéutica en el tumor vesical, sin querer sustituir a la cistoscopia tradicional, nos puede ayudar en el manejo de estos pacientes. Asimismo, es necesario realizar un seguimiento de estos resultados para conocer el impacto real a largo plazo, a nivel de recurrencias y progresión tumoral.

\section{CONCLUSIONES}

Nuestros resultados sugieren un incremento pacientes y $\mathrm{pT} 1$ en el $15 \%$ de los casos. Esta mejoría en las tasas de detección de tumores papilares se incrementó aún más en el caso concreto del carcinoma in situ $(56,8 \%)$, frente a la cistoscopia estándar. En nuestro caso, el $75 \%$ de los pacientes con carcinoma in situ se diagnosticaron mediante fluorescencia.

En este sentido, creemos que las mayores aportaciones que nos puede ofrecer la fotodinamia son el aumento del diagnóstico de pequeñas lesiones papilares, que si bien no van a cambiar la conducta a corto plazo en el tratamiento, si podrían significar una disminución en la tasa de recurrencias; y por otro lado, la mayor detección del carcinoma in situ, lesión potencialmente agresiva y con un alto riesgo de progresión tumoral.

Uno de los "inconvenientes" del diagnóstico fotodinámico es la alta tasa de falsos positivos. Estas oscilan entre el $13 \%$ (18) y el 59\% (19). Analizándolo en función de la lesión, obtendríamos un $40 \%$ para tumores papilares (20) y entre el $13-40 \%$ para el carcinoma in situ (21). En nuestra serie el porcentaje de pacientes con biopsias negativas bajo fluorescencia fue del $47 \%$. Están descritas situaciones en que podemos detectar áreas falsamente fluorescentes: edema por inflamación aguda, quimioterápico endovesical reciente (menos de 6 semanas), trabéculas vesicales, orificio de los meatos ureterales, divertículos.

En nuestro trabajo, se excluyeron los pacientes con BCG en las 6 semanas previas, pero probablemente, el porcentaje bajo de pacientes con citologías positivas $(20 \%)$, aumentó la posibilidad de biopsiar lesiones benignas con la fluorescencia. En cualquier caso, creemos que el beneficio para el paciente puede ser mayor, siendo agresivos con la toma de biopsias en zonas sospechosas a pesar de los falsos positivos. Además la cistoscopia blanca tampoco

\section{BIBLIOGRAFÍA Y LECTURAS \\ RECOMENDADAS (*lectura de interés $y^{* *}$ lectura fundamental)}

1. NATIONAL CANCER INSTITUTE OF THE NATIONAL INSTITUTES OF HEALTH.: "PDQ screening and prevention for patients: Screening for bladder cancer". http://cancernet.nci.nih.gov/ clinpdq/soa /Bladder_cancer_P.html. 1999.

2. BRAY, F.; SANKILA, R.; FERLAY, J. y cols.: "Estimates of cancer incidence and mortality in Europe in 1995". Eur. J. Cancer., 38: 99, 2002.

3. HERR, H.W.: "Natural history of superficial bladder tumors: 10 to 20 year follow up of treated patients". World J. Urol., 15: 84, 1997.

**4. JOCHAM, D.; STEPP, H.; WAIDELICH, R.: "Photodynamic diagnosis in Urology: State of the art". Eur. Urol., 2008 (pendiente de publicación).

5. WHITMORE, W.F. Jr.; BUSH, I.M.; ESQUIVEL, E.: "Tetracycline ultraviolet fluorescence in bladder carcinoma". Cancer, 17: 1528, 1964.

6. VICENTE, J.; CHECHILE, G.; ALGABA, F.: "Value of in vivo Mucosa-Staining Test with methylene blue in the diagnosis of pretumoral and tumoral lesions of the bladder". Eur. Urol., 13: 15, 1987. 
7. BENSON, R.C.; FARROW, G.M.; KINSEY, J.H. y cols.: "Detection and localization of in situ carcinoma of the bladder with hematoporphyrin derivate". Mayo Clin. Proc., 57: 548, 1982.

8. KRIEGMAIR, M.; BAUMGARTNER, R.; KNÜCHEL, R. y cols.: "Fluorescence photodetection of neoplastic urothelial lesions following intravesical instillation of 5-aminolevulinic acid". Urology, 44: 836, 1994.

9. BATTLE, A.M.: "Porphyrins, porphyries, cancer and photodynamic therapy, a model for carcinogenesis". J. Photochem. Photobiol. B., 20: 5, 1993.

*10. DANILTCHENKO, D.I.; RIEDL, C.R.; SACHS, M.D. y cols.: "Long-term Benedit of 5-aminolevulinic acid fluorescente assisted transurethral resection of superficial bladder cancer: 5 year results of a prospective randomized study". J. Urol., 174: 2129, 2005.

**11. DENZINGER, S.; BURGER, M.; WALTER, B. y cols.: "Clinically relevant reduction in risk of recurrence of superficial bladder cancer using 5aminolevulinic acid-induced fluorescente diagnosis: 8 year results of prospective randomized study". Urology, 69: 675, 2007.

12. D'HALLEWIN, M.A.; KAMUHABWA, A.R.; ROSKAMS, T. y cols.: "Hypericin-based fluorescence diagnosis of bladder carcinoma". BJU Int., 89: 760, 2002.

13. SIM, H.G.; LAU, W.; OLIVO, M. y cols.: "Is photodynamic diagnosis using hypericin better than white-light cystoscopy for detecting superficial bladder carcinoma?”. BJU Int., 95: 1215, 2005.

14. FOTITOS, N.; CAMPO, M.A.; POPOWYCZ, F. y cols.: "5- Aminolevulinic acid derivates in photomedicine: characteristics, application and perspectives". Photochem. Photobiol., 82: 994, 2006.

15. ABASCAL, R.: "Diagnóstico por fluorescencia en tumores vesicales". Arch. Esp. Urol., 60: 495, 2007.

16. ZAAK, D.; KRIEGMAIR, M.; STEPP, H. y cols.: "Endoscopic detection of transitional cell carcinoma with 5-aminolevulinic acid: Results of 1012 fluorescence endoscopies". Urology, 57: 590, 2001.

17. JICHLINSKI, P.; GUILLOU, L.; KARLSEN, S.J. y cols.: "Hexyl aminolevulinate fluorescence cystoscopy: A new diagnostic tool for the photodiagnosis of superficial bladder cancer, a multicentre study". J. Urol., 170: 226, 2003.

18. SCHMIDBAUER, J.;WITJES, F.; SCHMELLER, N. y cols.: "Improved detection of urothelial carcinoma in situ with hexaminolevulinate fluorescence cystoscopy". J. Urol., 171: 135, 2004.

*19. JOCHAM, D.; WITJES, F.; WAGNER, S. y cols.: "Improved detection and treatment of bladder cancer using hexaminolevulinate imaging: A prospective, phase III multicenter study". J. Urol., 174: 862, 2005.
*20. GROSSMAN, H.B.; GOMILLA, L.; FRADET, Y. y cols.: "A phase III, multicenter comparison of hexaminolevulinate fluorescente cystoscopy and white cystoscopy for the detection of superficial papillary lesions in patients with bladder cancer". J. Urol., 178: 62, 2007.

*21. FRADET, Y.; GROSSMAN, H.B.; GOMELLA, L. y cols.: "A phase III, multicenter comparison of hexaminolevulinate fluorescente cystoscopy and white cystoscopy for the detection of carcinoma in situ in patients with bladder cancer". J. Urol., 178: 68, 2007.

22. VAN DER MEIJDEN, A.; OESTERLINCK, W.; BRAUSI, M. y cols.: "Significance of bladder biopsias in Ta, T1 bladder tumors: A report from the EORTC Genito-Urinary Tract Cancer Cooperative group". Eur. Urol., 35: 267, 1999.

\section{COMENTARIO EDITORIAL}

El diagnóstico y tratamiento del tumor vesical superficial o "cáncer de vejiga que no infiltra músculo" es una de las situaciones más inquietantes en la práctica urológica al enfrentarnos a las múltiples posibilidades de evolución tumoral y en consecuencia con posibilidades terapéuticas que pasan de la vigilancia a la cistectomía.

Cualquiera que sea el método que mejore las opciones de diagnóstico y en consecuencia las de tratamiento deben ser valoradas y ofertadas al paciente o cuando menos puestas en su conocimiento sin entrar en discusiones de "rentabilidad económi$\mathrm{ca}^{\prime \prime}$ gerencial que disminuyan las opciones médicas.

Enfrentándonos al tumor vesical que no infiltra músculo en cuanto al riesgo de los pacientes, nos preocupa la recurrencia y la progresión. En el caso del presente trabajo, teniendo en cuenta que las lesiones incompletamentamente resecadas junto a aquellas que no se resecan por pasar inadvertidas, son la causa mas frecuente de recurrencia y que los pacientes que tienen una alta tasa de recurrencia tienen un mayor riesgo de progresión, independientemente del grado, este tipo de técnica tiene un papel preponderante al conseguir según la bibliografía seleccionada, incrementos en la detección del Cis. entre un 40 y $57 \%$ en relación a la cistoscopia convencional; asimismo refieren que "a un 20,5\% de los pacientes se les resecó al menos una lesión más de las que hubiésemos obtenido con la reseccion estándar de luz blanca", porcentaje nada desdeñable en cuanto a futuras recurrencias por ausencia de diagnóstico de lesiones inadvertidas en la cistoscopia convencional. 
Mas complejidad tiene la cistoscopia de control posterior a la instilación endovesical de inmuno-quimioterapia, cuya rentabilidad diagnóstica de diagnóstico tumoral se incrementa en relación a la experiencia y al número de biopsias de áreas sospechosas, en esta situación la alta tasa de falsos positivos comunicados en las distintas series (13 al 59\%, $47 \%$ en el trabajo comentado) nos deben hacer ser más cautos en su indicación.

En nuestra unidad que, con una mínima experiencia en el uso de Hexvix ${ }^{\circledR}$, tenemos una gran inquietud en cuanto al diagnóstico precoz de los tumores G-Ill y Cis., encontramos muy costosa la técnica ya que al coste del fármaco, hay que añadir el gasto que supone la adquisición de todo el sistema fotodinámico (óptica, fuente de luz, sistema de endovisión y ureteroscopio si se extiende la técnica al tracto urinario superior). En mi criterio, sería interesante aquilatar estos costes por parte de la industria de farmacia y endoscópica de tal forma que el desarrollo del diagnóstico fotodinámico no fracase por motivos económicos privando al paciente del gran beneficio de un diagnóstico precoz.

Una vez más, mi más sincera felicitación para el Dr. R. Abascal y todo su equipo por su constante aportación de mejoras a la Urología Asturiana en su quehacer diario y a la Española facilitándonos su enriquecedora experiencia.

José Ignacio Iglesia Prieto. Unidad de Urología. Clínica La Luz. Madrid. España. 